\title{
Investigación fenomenológica basada en la utilización de dispositivos móviles en la sala de clase
}

\author{
Phenomenological research based on the use of mobile devices in the classroom
}

Pesquisa fenomenológica baseada no uso de dispositivos móveis em sala de aula

\author{
ABNER JOSÉ COLÓN ORTIZ ${ }^{1}$ \\ IRIS NELLY RAMOS RULLÁN²
}

\begin{abstract}
RESUMEN
El presente artículo es una investigación cualitativa con un diseño fenomenológico sobre el uso de los dispositivos móviles en clases. El propósito de la investigación fue conocer el uso que les dan los estudiantes universitarios a los dispositivos móviles en la sala de clases. Se utilizaron como instrumentos una guía de preguntas para cuatro entrevistas semiestructuradas y dos grupos focales. El total de participantes fue de 14 estudiantes de nivel subgraduado y graduado de universidades del área sur de Puerto Rico. Se analizaron los resultados de las entrevistas utilizando el programa Atlas.ti 8. Luego de la codificación y categorización de las entrevistas y grupos focales, se concluye que los estudiantes utilizan los dispositivos móviles como una herramienta educativa útil de búsqueda de información, que en ocasiones suele ser un distractor en el proceso de enseñanza-aprendizaje y que debe regularse su uso en la sala de clases.
\end{abstract}

Palabras clave: Educación superior; entrevistas; grupo focal.

\begin{abstract}
This article is a qualitative investigation with a phenomenological design about the use of mobile devices in class. The purpose of the research was to know how university students use mobile devices in the classroom. A question guide for four semi-structured interviews and two focus groups were used as instruments. The total number of participants were fourteen undergraduate and graduate students from universities in the southern area of Puerto Rico. The results of the interviews were analyzed using the ATLAS.ti 8 program. After the coding and categorization of interviews and focus groups, it is concluded that students use mobile devices as a useful educational tool for information search, which is sometimes a distraction in the teaching-learning process and must be regulated its use in the classroom.
\end{abstract}

Keywords: Higher education; interviews; focus group. 


\section{RESUMO}

Este artigo é uma investigação qualitativa com um design fenomenológico sobre o uso de dispositivos móveis em sala de aula. O objetivo da pesquisa foi saber como os estudantes universitários usam dispositivos móveis na sala de aula. Um guia de perguntas para quatro entrevistas semiestruturadas e dois grupos focais foram utilizados como instrumentos. $\mathrm{O}$ número total de participantes foi de quatorze estudantes de graduação e pós-graduação de universidades da região sul de Porto Rico. Os resultados das entrevistas foram analisados no programa ATLAS.ti 8. Após a codificação e categorização das entrevistas e dos grupos focais, conclui-se que os alunos usam dispositivos móveis como uma ferramenta educacional útil para a busca de informações, o que às vezes é uma distração no processo de ensino-aprendizagem e deve ser regulamentado seu uso na sala de aula.

Palavras-chave: Ensino superior; entrevistas; grupo focal.

\section{INTRODUCCIÓN}

Los dispositivos móviles con acceso a internet brindan una infinidad de servicios y se han convertido en prácticamente una necesidad en muchos aspectos de la vida cotidiana, incluyendo la educación. En las universidades los teléfonos inteligentes, las tabletas y las computadoras portátiles forman parte habitual de la sala de clases, en la que los estudiantes consultan información en la internet y toman notas mientras el profesor explica; uso que se combina con grupos informales, mediante aplicaciones en los que los estudiantes intercambian más información entre ellos, que en los foros establecidos en los canales oficiales (Escobar, Glasserman \& Ramírez, 2015).

La inserción elevada de las tecnologías en general, y específicamente los dispositivos móviles digitales nos ofrecen multitud de servicios tales como la comunicación verbal y escrita, acceso a internet, sistema de cómputos, entre otros, que pueden abrir posibilidades a los profesores y estudiantes como herramientas de enseñanza-aprendizaje. Esta situación se ha convertido en un desafío para las instituciones educativas que debe responder a la sociedad e implementar estos recursos de manera eficiente en su acción formativa apoyando las metas pedagógicas (Fombona \& Rodil, 2018).

En esta investigación se pretendió entender el uso del dispositivo móvil en la sala de clases desde la perspectiva de los estudiantes universitarios y como se vincula en el proceso de enseñanza aprendizaje con el modelo de Conocimiento Tecnológico y Pedagógico del Contenido (TPACK), por sus siglas en inglés, de Koehler \& Mirsha (2005).

El modelo del Conocimiento Tecnológico y Pedagógico del Contenido, en inglés, Technological Pedagogical and Content Knowledge (TPACK), es un marco teórico conceptual del conocimiento desarrollado por Koehler \& Mishra (2005). Este modelo presenta la interacción entre el conocimiento $(\mathrm{K})$ de la tecnología $(\mathrm{T})$, pedagogía $(\mathrm{P})$ y el contenido $(\mathrm{C})$ de una disciplina y la interrelación entre ellos (Mishra \& Koehler, 2006).

La base del marco teórico de TPACK se inicia con el modelo Conocimiento Contenido Pedagógico desarrollado por Shulman, Pedagogical Content Knowledge, por sus siglas en inglés PCK (Mishra \& Koehler, 2006). En el modelo PCK, Shulman reconoció la necesidad 
de un marco teórico que relacionara el conocimiento de contenido que poseen los docentes, con buenas prácticas de enseñanza. Mishra \& Koehler (2006) integraron la dimensión de tecnología al modelo PCK y como resultado, desarrollaron el modelo TPACK combinando el conocimiento del contenido y pedagogía con la tecnología y definiendo el modelo como la interacción de estos tres tipos de conocimientos.

En el modelo TPACK, Koehler \& Mishra (2005) presentan siete componentes: (a) conocimiento tecnológico (TK), (b) conocimiento de contenido (CK), (c) conocimiento pedagógico (PK), (d) conocimiento de contenido pedagógico (TPK), (e) conocimiento de contenido tecnológico (TCK), (f) conocimiento pedagógico tecnológico (TPK), y (g) conocimiento tecnológico pedagógico del contenido (TPCK).

Según Koehler \& Mishra, el área de contenido (C) es la materia que se va a aprender o a enseñar. El área de tecnología (T) incluyen las tecnologías actuales tales como: (a) computadoras, (b) internet, (c) videos digitales, (d) libros, y (e) proyectores. El área de pedagogía (P) describe las prácticas, procesos, estrategias, métodos de enseñanza, avalúo y aprendizaje del estudiante (Koehler \& Mishra, 2005).

La importancia de la aplicación del modelo TPACK, es que los docentes deben comprender la manera en que los elementos que componen el mismo se relacionan para apoyar las metas pedagógicas utilizando la tecnología. En el caso de esta investigación "El uso de los dispositivos móviles en la sala de clases", se inserta en el modelo TPACK, el uso de los dispositivos móviles (MD), Mobile Devices en inglés, en las áreas tecnológicas del modelo: conocimiento tecnológico (TK), conocimiento pedagógico tecnológico (TPK), conocimiento tecnológico del contenido (TCK) y en el centro del modelo TPACK que integra el conocimiento pedagógico y tecnológico del contenido.

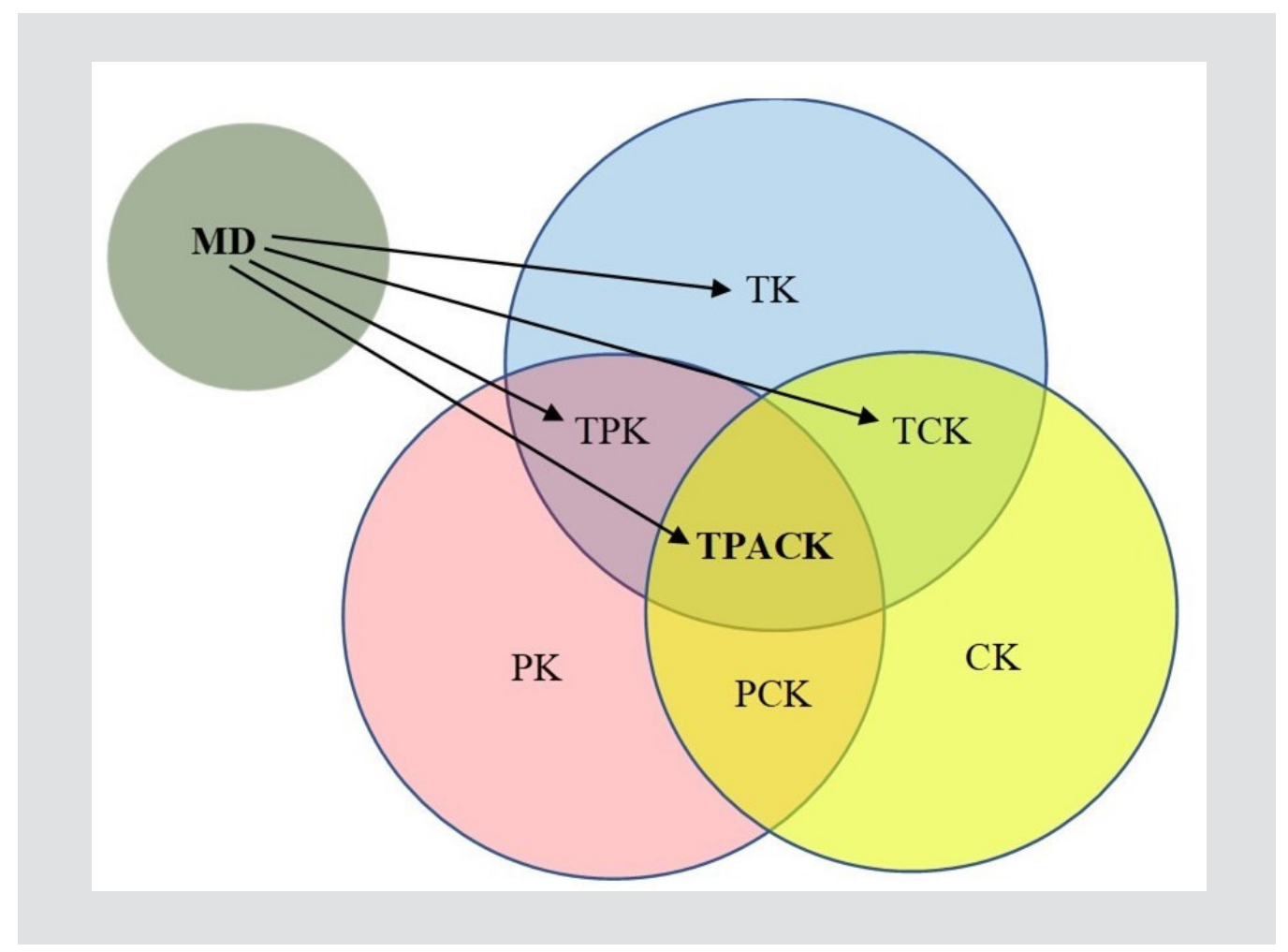

Figura 1. El uso de los dispositivos móviles (MD) en el modelo TPACK 
En un estudio realizado en el oeste de los Estados Unidos de América a 442 estudiantes de escuela intermedia, se pretendió investigar las percepciones de los estudiantes y el uso real de los dispositivos móviles en la sala de clases (Bartholomew \& Reeve, 2018). En este estudio se les administró a los estudiantes un cuestionario para conocer los posibles usos del dispositivo móvil en la sala de clases. Luego se dividieron los estudiantes en dos grupos, a la mitad se les permitió utilizar el dispositivo móvil en una unidad de estudio de dos semanas y a la otra mitad no se les permitió utilizar el celular en la misma unidad de estudio por dos semanas. Según Bartholomew \& Reeve (2018) algunos de los resultados obtenidos demuestran que hay varias brechas entre las percepciones de cómo se usarían los dispositivos móviles y el uso real que surgió por parte de los estudiantes. Los estudiantes no utilizaron los dispositivos móviles tan a menudo como lo planificaron. Por otra parte, los investigadores concluyeron que los dispositivos móviles en la sala de clases de $\mathrm{K}$ a 12 puede ser perjudicial para el rendimiento estudiantil (Bartholomew \& Reeve, 2018).

Fombona y Rodil (2018) realizaron un estudio para determinar el grado de implementación real y de aceptación de los dispositivos móviles en los distintos niveles de enseñanza en un centro educativo español. Esta investigación evidencia que profesores y estudiantes poseen dispositivos móviles, pero la mitad afirma que no los utiliza como herramienta de enseñanza. La mitad de los profesores dudan que pueda mejorar la educación tradicional, aunque a la mayoría les gustaría implementarlo y piensan que es un factor motivador para los estudiantes. Sin embargo, los estudiantes muestran mayor interés por el uso de los dispositivos móviles y piensan que tendrían mejor rendimiento académico. Los investigadores concluyen que los dispositivos móviles tienen un uso reducido como herramienta de enseñanza y aprendizaje en la sala de clases, a pesar de que a la gran mayoría de los profesores y estudiantes les gustaría poder utilizarlo más a menudo (Fombona \& Rodil, 2018).

Jackson (2013) llevó a cabo una investigación que describe las percepciones de los estudiantes universitarios con respecto al uso de los dispositivos móviles en la sala de clases. Se encuestaron 102 estudiantes subgraduados de una Universidad de California con una edad promedio de 18.5 años. Los principales hallazgos de este estudio incluyen: (a) el 76\% de los participantes perciben el dispositivo móvil como un distractor, mientras que las computadoras portátiles son percibidas por el 90\% como una herramienta de aprendizaje útil, (b) que los estudiantes quisieran que los profesores los consideraran cuando redactaran las políticas relacionadas al uso de la tecnología móvil, (c) la mayoría de los estudiantes piensan que los profesores deben articular las políticas, (d) aproximadamente la mitad de los estudiantes son conscientes de las formas en que la tecnología móvil es utilizada para hacer trampas y la mayoría de los estudiantes se sienten molestos por las prohibiciones completas del uso de la tecnología móvil en la sala de clases. Jackson (2013) concluyó que las perspectivas tanto de los estudiantes como de los docentes son importantes para entender los beneficios y dificultades que plantean los dispositivos móviles en entornos educativos. Los docentes que han sido conscientes de antemano sobre sus políticas, y que han educado a sus estudiantes con respecto a los usos esperados, contribuirán a un entorno de aprendizaje más reflexivo y funcional (Jackson, 2013).

Navarro, Guzmán \& García (2019), realizaron una investigación titulada: La integración tecnológica en el aula, significaciones desde estudiantes de educación secundaria. La investigación de estos autores tuvo como objetivo, destacar las significaciones y atribuciones que 
los estudiantes de secundaria establecen con relación a la integración tecnológica en la sala de clases y los procesos de aprendizaje facilitados por dicha tecnología. Los participantes fueron 128 estudiantes entrevistados. Los resultados muestran tres niveles de la integración de la tecnología en la sala de clases: (1) aprendizaje móvil y medios tradicionales, (2) aprendizaje móvil y tecnologías audiovisuales y (3) tecnologías audiovisuales y medios tradicionales. Los investigadores concluyeron que se resaltan más atribuciones favorables a la tecnología en la sala de clases que resistencias y que los estudiantes demandan una fuerte conectividad de diversas aplicaciones educativas en la sala de clases (Navarro, Guzmán \& García, 2019).

En otra investigación realizada por Maldonado, Balladares \& Rivas (2019) se determinó la percepción de los profesores y estudiantes respecto al uso educativo de los dispositivos móviles. El 75\% de los participantes asociaron el uso de los dispositivos móviles con el aprendizaje y que este aprendizaje se adquirió de forma divertida. Los investigadores concluyen que el dispositivo móvil (tableta) les permite a los actores educativos acceder a información, conocer diferentes culturas y les posibilita la investigación generando un aprendizaje significativo de manera positiva para el proceso de enseñanza (Maldonado, Balladares \& Rivas, 2019).

Oliva, (2014) realizó un estudio en 69 instituciones educativas de los 14 departamentos de El Salvador. Los participantes fueron 1,384 estudiantes de escuela secundaria y 200 docentes de distintos partes del país. El propósito principal del estudio fue conocer si el uso del dispositivo móvil es un recurso didáctico de uso pedagógico o un distractor que genera problemas entre profesores y estudiantes debido al uso dentro de la sala de clases. En los resultados se obtuvo que el uso de los dispositivos móviles (celulares) en la sala de clases está afectando el aprendizaje de los estudiantes, ya que estos muestran dispersión, falta de concentración, actitud poco reflexiva y crítica, baja calidad de las actividades y, como consecuencia de esto, un bajo rendimiento escolar. Por otro lado, se obtuvo una tendencia a enfocar al celular como el principal enemigo actual del aprendizaje, pues su uso inadecuado interfiere en el rendimiento académico, ya que los estudiantes prefieren dar prioridad a la tecnología de vanguardia de su dispositivo móvil que a lo que se explica en clases. Oliva, (2014) concluye que se debe diseñar una estrategia didáctica para integrar el uso de los dispositivos móviles (celulares) como un medio alfabetizador funcional para tecnologías.

\section{METODOLOGÍA}

Según Creswell (2011) en un diseño fenomenológico se pretende entender y describir los fenómenos desde el punto de vista de cada participante y de la percepción colectiva. El diseño utilizado en esta investigación cualitativa fue un diseño fenomenológico debido a que el interés primario de la investigación fue describir y entender el uso de los dispositivos móviles en la sala de clases desde el punto de vista de las experiencias de los estudiantes. El escenario de investigación fueron universidades del área sur de Puerto Rico.

Los participantes de esta investigación fueron 4 estudiantes subgraduados y 10 estudiantes graduados residentes de Puerto Rico. Los criterios incluyentes fueron los siguientes: estar matriculados en una universidad en Puerto Rico y haber aceptado participar de la investigación ya sea mediante entrevista individual o grupo focal. Las edades de los participantes fluctuaron entre 21 a 45 años. 
La información se recopiló mediante 4 entrevistas semiestructuradas y 2 grupos focales. Los participantes de las entrevistas individuales optaron por reunirse en espacios fuera de la universidad, pero el investigador se aseguró que los mismos cumplieran con los requisitos de accesibilidad, privacidad, confidencialidad y disponibilidad por el tiempo necesario. Estos lugares tenían las condiciones adecuadas para que se cumpliera con los requerimientos éticos de la investigación.

Se utilizó un protocolo de preguntas semiestructuradas. Esta guía de preguntas fue revisada por un grupo de expertos que colaboró en su edición al aportar consistencia y rigurosidad al proceso de entrevista. Además, se formularon preguntas cognitivas para la validez de las interpretaciones en las preguntas realizadas en las entrevistas. La idea es entender por qué una persona responde como lo hace y determinar el modo en que una pregunta se comportará, es decir cuál es el valor interpretativo de esta (Fowler, Lloyd, Cosenza \& Wilson, 2014). El protocolo permitió hacer preguntas adicionales que fueron meritorias para la investigación. Las entrevistas fueron grabadas en forma digital, según previa autorización de los participantes y transcritas adverbatin. Las secciones de las entrevistas se programaron de 50 a 90 minutos que corresponde a un modo de carácter extenso, según sugerido por Lucca \& Berrios (2009) para las entrevistas semiestructuradas. En la tabla 1 se describen los participantes, el tiempo y las fechas de las entrevistas.

Tabla 1

Entrevistas realizadas a los participantes

\begin{tabular}{|c|c|c|c|}
\hline PARTICIPANTES & NIVEL & TIEMPO & FECHA \\
\hline Estudiante 1 & Subgraduado & 60 minutos & 13 de abril de 2019 \\
\hline Estudiante 2 & Subgraduado & 80 minutos & 13 de abril de 2019 \\
\hline Estudiante 3 & Subgraduado & 65 minutos & 13 de abril de 2019 \\
\hline Estudiante 4 & Subgraduado & 60 minutos & 6 de mayo de 2019 \\
\hline
\end{tabular}

Los grupos focales se llevaron a cabo en una universidad privada del área sur de Puerto Rico. El grupo focal 1 estuvo constituido por 6 estudiantes graduados y el grupo focal 2 por 4 estudiantes graduados, según sugerido por Creswell (2011). Las secciones de las entrevistas se programaron de 50 a 90 minutos que corresponde a un modo de carácter extenso, según sugerido por Lucca \& Berrios (2009) para las entrevistas semiestructuradas.

En la tabla 2 se describen los participantes por grupo focal, el tiempo y las fechas de las entrevistas. La información que surgió fue de conocimiento de todos los participantes de cada grupo por separado. A estos se les instruyó para que no mencionaran sus nombres durante el desarrollo del grupo focal correspondiente. Cada participante del grupo fue identificado con un número con el propósito de facilitar su intervención en la entrevista. Los participantes tenían que mencionar el número asignado antes de expresar algún comentario o contestación a las preguntas. Esto evitó que los participantes quedaran registrados en la grabación, al asegurar así la confiabilidad de los mismos. 
Tabla 2

Grupos focales

\begin{tabular}{|c|c|c|c|}
\hline PARTICIPANTES GRUPOS FOCALES & NIVEL & NIVEL & FECHA \\
\hline Grupo focal 1 (6 parti-cipantes) & Graduado & Graduado & 23 de abril de 2019 \\
\hline Grupo focal 2 (4 parti-cipantes) & Graduado & Graduado & 30 de abril de 2019 \\
\hline
\end{tabular}

Para el análisis cualitativo de la información obtenida mediante las entrevistas semiestructuradas y los grupos focales, se realizó primeramente la transcripción adverbatin de las entrevistas grabadas. Estas transcripciones se codificaron y categorizaron utilizando el programado Atlas.ti versión 8.0. En la tabla 3 se organizan la relación de las preguntas de investigación con la estrategia de recopilación y el análisis correspondiente.

Tabla 3

Relación de las preguntas de investigación con la estrategia de recopilación y el análisis de la información.

\section{PREGUNTAS}

¿Cuál es el uso de los dispositivos móviles en la sala de clases desde el punto de vista del estudiante?

¿Qué usos educativos tiene el dispositivo móvil en la sala de clases?

¿En qué forma el dispositivo móvil le afecta o beneficia al estudiante en su desempeño académico?

¿De qué manera el dispositivo móvil se vincula con el proceso de enseñanza aprendizaje?

\section{TÉCNICA O ESTRATEGIA}

Entrevista semiestructurada

Grupo focal

Entrevista semiestructurada

Grupo focal

Entrevista semiestructurada

Grupo focal

Entrevista semiestructurada

Grupo focal

\author{
ANÁLISIS \\ Transcripción \\ Codificación y categorización \\ con el programado Atlas.ti 8 \\ Transcripción \\ Codificación y categorización \\ con el programado Atlas.ti 8 \\ Transcripción \\ Codificación y categorización \\ con el programado Atlas.ti 8 \\ Transcripción \\ Codificación y categorización \\ con el programado Atlas.ti 8
}

\section{RESULTADOS}

Para el análisis de la información se realizó la transcripción de las preguntas adverbatin. Se utilizó el programa Atlas.ti 8 para codificar y categorizar la información de las entrevistas y los grupos focales. Se identificaron patrones en las respuestas que pudieron agruparse en códigos y categorizarse para poder realizar un análisis del fenómeno (ver figura 1). Se identificaron tres categorías del uso de los dispositivos móviles: (1) herramienta, (2) regulación y (3) distractor. Estas categorías surgieron de unos patrones que se identificaron luego de realizar la transcripción. En la figura 2 se muestra la red de categorías y códigos que se generaron luego del análisis de la información utilizando el programado Atlas.ti 8. 
Para la categoría "herramienta" se identificaron los siguientes códigos: buscador, comunicación, útil, aprendizaje, información y proceso de enseñanza. Por otro lado, para la categoría "regulación" se identificaron los siguientes códigos: aprendizaje, proceso de enseñanza, tiempo de uso y pérdida de tiempo. Los códigos identificados para la categoría "distractor" fueron los siguientes: información, proceso de enseñanza y redes sociales. Según los resultados existen varios códigos que se repiten en distintas categorías como lo es "proceso de enseñanza" que se codifica para las tres categorías ya que en varias citas se puede interpretar que los participantes perciben que el uso del dispositivo móvil en el proceso de enseñanza es una herramienta útil, que en ocasiones resulta ser un distractor y debe regularse.

Para la categoría "herramienta", se citan adverbatin algunas de las opiniones de los participantes: "el uso del dispositivo móvil dentro del salón de clases, es que tienes acceso a información rápida", "puede ser una herramienta, se puede utilizar para obtener información adicional", "me parece que la integración de dispositivos móviles provee una visión distinta del aprendizaje en la sala de clases", "entiendo que es una herramienta que aporta al proceso de enseñanza-aprendizaje", "el uso de dispositivos móviles en la sala de clases aporta al proceso de aprendizaje, puesto que nos da la oportunidad de profundizar en el contenido o información e incluso buscar ejemplos que pueden ayudar a entender el tema de discusión".

Para la categoría "regulación" se citan las siguientes expresiones: "pueden desviar la atención del estudiante, si no se tiene un buen control o no se explica bien el uso que se le va a dar, en cierto momento, se puede perder la esencia o la función de integrarlos en la sala de clases", "pienso que sí se debe regular, muchas veces o en la mayoría de las veces los utilizan para otros propósitos no educativos".

Para la categoría "distractor" se citan las siguientes opiniones "el uso inadecuado del celular en la sala de clases puede distraer si no hay buen control", "estoy de acuerdo con la compañera, cuando se convierte en un distractor es cuando no hay supervisión de parte de la persona que está impartiendo la clase o el curso", "...y aunque no lo tengo sonando y al ver la cantidad de mensajes uno tiende a contestar y eso puede ser un distractor".

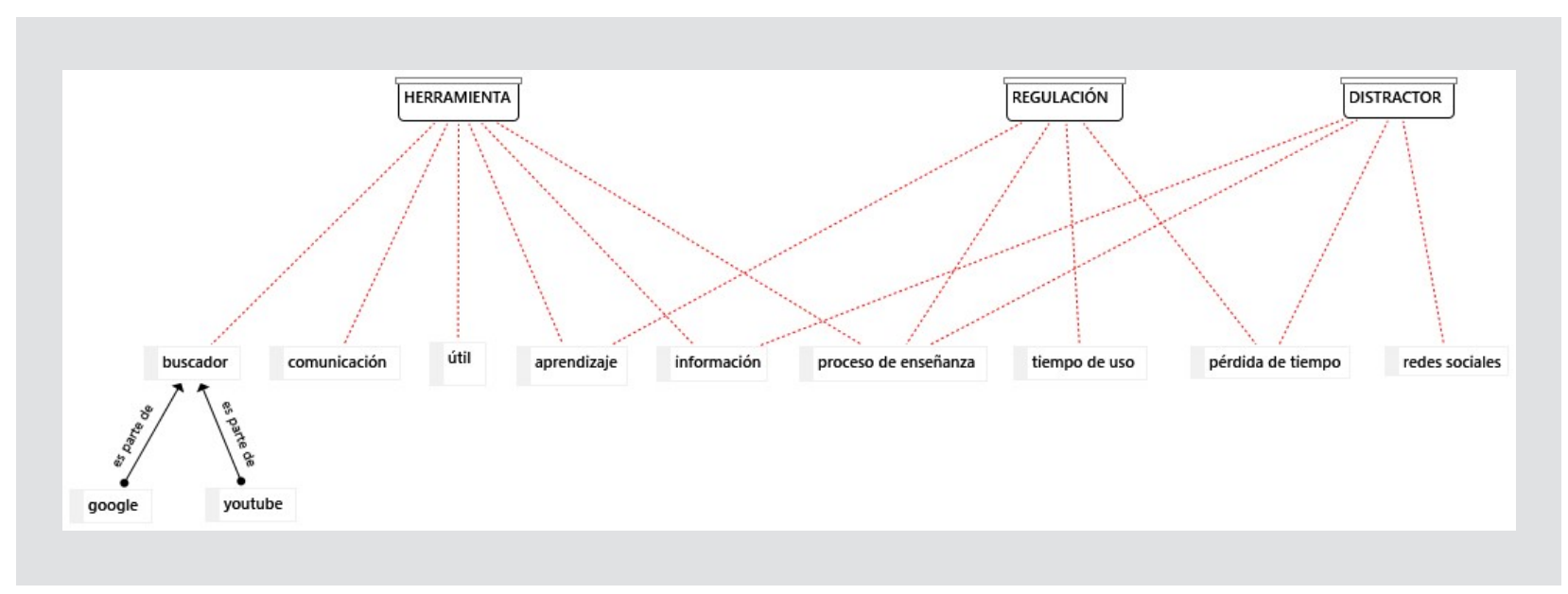

Figura 2. Resultados del uso de los dispositivos móviles en la sala de clases 
Desde el punto de vista de los estudiantes a nivel subgraduado y graduado de universidades del área sur de Puerto Rico, el dispositivo móvil en la sala de clases tiene como uso principal ser una herramienta útil en la búsqueda de información accesible y rápida. Entre los buscadores más utilizados por los participantes están Google y YouTube. Uno de los usos más comunes con la plataforma de la universidad, es bajar las presentaciones de los cursos y tener comunicación con los profesores mediante correo electrónico. Un participante comentó: "los profesores ponen lo que van a dar en las clases y nosotros lo bajamos y lo vemos... así, si pasa la diapositiva rápido... yo lo puedo ver en mi celular o computadora". Esto converge con Jackson (2013), que afirmó que una de las percepciones positivas de los estudiantes a nivel subgraduado es el acceso al material de la clase y presentaciones en PowerPoint. Según Maldonado, Balladares \& Rivas (2019), el dispositivo móvil permite a los estudiantes y profesores acceder a más información posibilitando la investigación y generando un aprendizaje más significativo. Por tanto, se observa que el uso del dispositivo móvil en la sala de clases es percibido como una herramienta conveniente que facilita la búsqueda de información.

Por una parte, se vinculó el modelo Conocimiento Tecnológico Pedagógico del Contenido, en inglés, Technological Pedagogical Content Knowledge, (TPACK) con la utilización de los dispositivos móviles, específicamente en el uso que le dan los profesores para enviar información a través de plataformas universitarias tales como Moodle y mediante correos electrónicos, así como la integración de videos de YouTube en el proceso de enseñanza aprendizaje y la búsqueda de información en Google. De esta forma, reconociendo la tendencia de integrar la tecnología en el proceso educativo, Mishra \& Koehler (2006) plantean en su modelo TPACK la importancia del conocimiento pedagógico del contenido que debe tener el docente para la integración de tecnologías en el proceso de enseñanza. Esto se vincula con Navarro, Guzmán \& García (2019) que concluyeron en su investigación que existe más atribuciones positivas que negativas hacia el uso de la tecnología en la sala de clases y que los estudiantes demandan una alta conectividad de diversas aplicaciones educativas en el proceso de enseñanza.

Por otra parte, en esta investigación un participante afirma que el uso de los dispositivos móviles le ayuda en su desempeño académico. Esto diverge de la investigación acerca de las percepciones de los estudiantes de nivel intermedio de Bartholomew \& Reeve (2018), que concluyeron que el uso de los dispositivos móviles es perjudicial para el desempeño de los estudiantes. Esto concuerda con la investigación de la integración tecnológica en el aula de Navarro, Guzmán \& García (2019) donde se significan más atribuciones favorables a la tecnología en la sala de clases que resistencias a la integración tecnológica. También, converge con la investigación referente a los niveles de uso y aceptación de los dispositivos móviles en el aula de Fombona \& Rodil (2018), que indicaron que los alumnos muestran un mayor interés y creen que el uso de los dispositivos móviles mejoraría su rendimiento.

Cabe destacar que algunos participantes mencionan que el uso del dispositivo móvil puede ser un distractor y que el mismo debe ser regulado. Algunas citas obtenidas de las entrevistas son las siguientes: "pienso que el dispositivo móvil dentro de la sala de clases debe ser regulado", "es una buena herramienta, bien dirigido", "lo veo positivo porque es una herramienta, pero a la vez que se utiliza para sustituir memoria y análisis, es un problema", "puede ser un distractor cuando se utiliza sin supervisión". Estas afirmaciones concuerdan 
con la investigación: El uso de teléfonos móviles en el sistema educativo público de El Salvador: ¿Recurso didáctico o distractor pedagógico?, en la cual se concluyó que la telefonía celular está afectando el aprendizaje de los estudiantes, ya que estos muestran dispersión, falta de concentración, actitud poco reflexiva y crítica, baja calidad de las actividades y un bajo rendimiento escolar (Oliva, 2014). También concuerdan con Jackson (2013) donde el 76\% de los participantes perciben el dispositivo móvil como un distractor. Por tanto, se observa como la utilización del dispositivo móvil en la sala de clases es percibido como un distractor tanto para los estudiantes a nivel de escuela secundaria como los estudiantes a nivel de educación superior.

\section{CONCLUSIONES}

El dispositivo móvil tiene diversidad de usos en la sala de clases. Basado en los resultados de esta investigación fenomenológica se concluye que las experiencias de los estudiantes universitarios es una mayormente positiva. Les ha permitido utilizarlo como una técnica útil en la sala de clases para la búsqueda rápida de la información. Además, les ha beneficiado en el desempeño académico gracias al acceso de la información y a las múltiples aplicaciones que utilizan tanto de manera personal como académica y en conjunto a sus profesores y compañeros de clases. Sin embargo, la mayoría de los participantes afirman que tener un dispositivo móvil en la sala de clases en ocasiones suele ser un distractor. Algunos participantes concuerdan que el uso del dispositivo móvil debe ser regulado en la sala de clases para que se use como técnica educativa y se minimice la posibilidad de que afecte negativamente el proceso de enseñanza y aprendizaje al convertirse en un posible distractor.

\section{REFERENCIAS}

Bartholomew, S. R., \& Reeve, E. (2018). Middle School Student Perceptions and Actual Use of Mobile Devices: Highlighting Disconnects in Student Planned and Actual Usage of Mobile Devices in Class. Educational Technology \& Society, 21(1), 4858.

Creswell, J. W. (2014). Research Design: Qualitative, Quantitative and Mixed Methods Approaches (4th ed.). Sage.

Escobar, J. V., Glasserman. L. D., \& Ramírez Montoya, M. S. (2015). Apropiación tecnológica con pizarrón interactivo y tabletas digitales en profesores de educación básica. EDUTEC. Revista Electrónica de Tecnología Educativa, 53, 1-15

Fombona, J. \& Rodil, F.J. (2018). Niveles de uso y aceptación de los dispositivos móviles en el aula. Revista de Medios y Educación, 52, 21-35.

Fowler, F. J., Lloyd, S. J., Cosenza, C. A. \& Wilson, I. B. (2014). Coding Cognitive Interview: an approach to enhancing the value of cognitive testing for survey question evaluation. Field Methods, 28(1) 1-18.

Jackson, L.D. (2013). Is Mobile Technology in the Classroom a Helpful Tool or a Distraction? A Report of University Students' Attitudes, Usage Practices, and Suggestions for Policies. The International Journal of Technology, Knowledge, and Society, 8(5), 129-140. 
Koehler, M.J. \& Mishra, P (2005). What happens when teachers design educational technology? The development of technological pedagogical content knowledge. Educational Computing Research, 32(2), 131-152.

Lucca-Irizarry, I. \& Berríos-Rivera, R. (2009). La investigación cualitativa: fundamentos, diseños y estrategias. Ediciones SM.

Maldonado-Garcés, V., Balladares-Burgos, J. \& Rivas -Toledo, A. (2019) Perception of educational actors about the use of mobile devices: A case study. Revista Cátedra, $2(3), 39-53$.

Mishra, P. \& Koehler, M. J. (2006). Technological Pedagogical Content Knowledge: A Framework for Teacher Knowledge. Teachers College Record, 108(6), 1017-1054.

Navarro-Rodríguez, M., Guzmán-Arredondo, A. \& García Arámbula, N. S. (2019). La integración tecnológica en el aula, significaciones desde estudiantes de educación secundaria. 3C TIC. Cuadernos de desarrollo aplicados a las TIC, 8(2), 70-83.

Oliva, H. A. (2014). El uso de teléfonos móviles en el sistema educativo público de El Salvador: ¿Recurso didáctico o distractor pedagógico? Realidad y reflexión, 40, 60-76. 